\title{
Graph Neural Networks for Graph Search
}

\author{
Yizhou Sun \\ Department of Computer Science, UCLA \\ yzsun@cs.ucla.edu
}

\begin{abstract}
Graph neural networks (GNNs) have received more and more attention in past several years, due to the wide applications of graphs and networks, and the superiority of their performance compared to traditional heuristics-driven approaches. However, most existing GNNs still focus on node-level applications, such as node classification and link prediction, and many challenging graph tasks are graph-level, such as graph search. In this talk, I will introduce our recent progress on graph-level neural operator development. In particular, we will examine three challenging tasks that are key to the success of graph search: (1) How can we conduct efficient graph similarity search by turning the NP-Complete problems, such as the Graph Edit Distance (GED) and Maximum Common Subgraph (MCS) computation, into a learning problem? We will present SimGNN [1] and GraphSim [3] that are able to provide more efficient and effective results compared to state-of-the-art approximate algorithms. (2) How can we provide a neural operator that can turn any graph into a low dimensional representation vector, which is learnable, inductive, and unsupervised? In this line, we propose UGraphEmd [2] that is able to leverage graph-graph interaction to produce manifold-preserving graph-level embedding. Moreover, GHashing [5] is designed to map each graph to a discrete hash code, which enables a much more efficient search (20 times speed up) to handle large graph database with millions of graphs. And (3) how can we design GNNs that can directly detect the best matched subgraphs of two graphs? A deep reinforcement learning framework RLMCS [4] is then proposed to address this issue, with the goal to learn the best strategy to pick the next matching pair for two graphs. In the end, we will provide some discussions to the open questions in the field.
\end{abstract}

\section{CCS CONCEPTS}

- Computing methodologies $\rightarrow$ Learning latent representations; • Information systems $\rightarrow$ Information systems applications.

\section{KEYWORDS}

graph search, graph neural networks, graph representation learning, graph edit distance, graph hashing, subgraph isomorphism

\section{ACM Reference Format:}

Yizhou Sun. 2020. Graph Neural Networks for Graph Search. In 3rd foint International Workshop on Graph Data Management Experiences Systems

Permission to make digital or hard copies of part or all of this work for personal or classroom use is granted without fee provided that copies are not made or distributed for profit or commercial advantage and that copies bear this notice and the full citation on the first page. Copyrights for third-party components of this work must be honored. For all other uses, contact the owner/author(s).

GRADES-NDA'20, June 14, 2020, Portland, OR, USA

(C) 2020 Copyright held by the owner/author(s).

ACM ISBN 978-1-4503-8021-8/20/06.

https://doi.org/10.1145/3398682.3399159
(GRADES) and Network Data Analytics (NDA) (GRADES-NDA'20), fune 14, 2020, Portland, OR, USA. ACM, New York, NY, USA, 1 page. https://doi.org/ $10.1145 / 3398682.3399159$

\section{ABOUT THE SPEAKER}

Yizhou Sun is an associate professor at department of computer science of UCLA. Prior to that, she was an assistant professor in the College of Computer and Information Science of Northeastern University. She received her Ph.D. in Computer Science from the University of Illinois at Urbana-Champaign in 2012. Her principal research interest is on mining graphs/networks, and more generally in data mining, machine learning, and network science, with a focus on modeling novel problems and proposing scalable algorithms for large-scale, real-world applications. She is a pioneer researcher in mining heterogeneous information network, with a recent focus on deep learning on graphs/networks. Yizhou has over 100 publications in books, journals, and major conferences. Tutorials of her research have been given in many premier conferences. She received 2012 ACM SIGKDD Best Student Paper Award, 2013 ACM SIGKDD Doctoral Dissertation Award, 2013 Yahoo ACE (Academic Career Enhancement) Award, 2015 NSF CAREER Award, 2016 CS@ILLINOIS Distinguished Educator Award, 2018 Amazon Research Award, and 2019 Okawa Foundation Research Grant.

\section{ACKNOWLEDGMENTS}

This work is partially supported by NSF III-1705169, NSF CAREER Award 1741634, NSF 1937599, DARPA HR00112090027, Okawa Foundation Grant, and Amazon Research Award.

This work is a collaboration with Wei Wang, Yunsheng Bai, Hao Ding, Derek Xu, Zongyue Qin, Ting Chen, Bian Song, Ken Gu, Alex Wang, Agustin Marinovic, and others.

\section{REFERENCES}

[1] Yunsheng Bai, Hao Ding, Song Bian, Ting Chen, Yizhou Sun, and Wei Wang. 2019. SimGNN: A Neural Network Approach to Fast Graph Similarity Computation. In Proceedings of the Twelfth ACM International Conference on Web Search and Data Mining (Melbourne VIC, Australia) (WSDM '19). Association for Computing Machinery, New York, NY, USA, 384-392. https://doi.org/10.1145/3289600.3290967

[2] Yunsheng Bai, Hao Ding, Yang Qiao, Agustin Marinovic, Ken Gu, Ting Chen, Yizhou Sun, and Wei Wang. 2019. Unsupervised Inductive Graph-Level Representation Learning via Graph-Graph Proximity. In Proc. of 2019 Int. foint Conf. on Artificial Intelligence (I7CAI'19) (Macao, China).

[3] Yunsheng Bai, Hao Ding, Yizhou Sun, and Wei Wang. 2020. Learning-based Efficient Graph Similarity Computation via Multi-Scale Convolutional Set Matching. In Proc. of the 34rd AAAI Conference on Artificial Intelligence (AAAI'20) (New York, New York).

[4] Yunsheng Bai, Derek Xu, Alex Wang, Ken Gu, Xueqing Wu, Agustin Marinovic, Christopher Ro, Yizhou Sun, and Wei Wang. 2020. Fast Detection of Maximum Common Subgraph via Deep Q-Learning. arXiv:2002.03129 [cs.LG]

[5] Zongyue Qin, Yunsheng Bai, and Yizhou Sun. 2020. GHashing: Semantic Graph Hashing for Approximate Similarity Search in Graph Databases. In Proc. of 2020 ACM SIGKDD Int. Conf. on Knowledge Discovery and Data Mining (KDD'20) (San Diego, CA). 